\title{
Concepciones y prácticas de los profesores sobre la evaluación de la oralidad
}

\author{
Conceptions and Practices of Teachers \\ on the Evaluation of Oral Language
}

\author{
Raquel Pinilla Vásquez* \\ María Elvira Rodríguez Luna \\ Yolima Gutiérrez Ríos \\ María Fernanda Torres \\ Martha Judith Camelo \\ Julia Esther Casjulia
}

Recibido: 16 de septiembre de 2012

Aceptado: 26 de octubre de 2012

\section{Resumen}

El artículo presenta los resultados finales de la investigación "Evaluar lo oral para cualificar las prácticas de enseñanza-aprendizaje de la lengua materna", cuyo objetivo central es identificar aspectos comunes con respecto a la evaluación de la lengua oral, específicamente en lo que se refiere a: ¿qué se evalúa?, ¿por qué y para qué?, ¿cómo se evalúa? y ¿con qué instrumentos? Se trata de una investigación de carácter interpretativo que permite analizar las concepciones y las prácticas de enseñanza y de evaluación de la lengua oral en instituciones de educación secundaria ubicadas en Bogotá D. C. Los resultados señalan los avances y limitaciones en la formación de docentes, indican las competencias docentes que requieren cualificación y, a su vez, aportan herramientas conceptuales y prácticas que posibilitan la adecuada recontextualización de los avances logrados por las ciencias del lenguaje y la pedagogía.

Palabras clave: evaluación, oralidad, lengua oral, concepciones, enseñanza, aprendizaje.

\section{Abstract}

The article presents the final results of the research "Oral language evaluation for the qualification on practices of teaching and learning the mother tongue". It's main objective is to identify the common aspects of the evaluation of the oral language, specifically around the questions: what is evaluated?, why and what for?, how is it evaluated?, what instruments are used in order to do so? The research has an interpretative character, allowing the analysis of the conceptions and practices of the evaluation and the teaching of the oral language in some secondary school educational institutions located in Bogota D.C. The results point to the advancements and limitations in the linguistic formation of teachers, also indicate the teaching competence that require qualification. The results as well present conceptual and practical tools that permit an adequate re-contextualization of the advancements achieved on the language and pedagogic sciences.

Key words: evaluation, oral language, orality, conceptions, teaching, learning.

* Las autoras son miembros del grupo de investigación Lenguaje, Cultura e Identidad de la Universidad Distrital Francisco José de Caldas y son responsables de la investigación "Evaluar lo oral para cualificar las prácticas de enseñanza-aprendizaje de la lengua materna" de la cual se deriva el presente artículo . 


\section{Introducción}

El proyecto "Evaluar lo oral para cualificar las prácticas de enseñanza-aprendizaje de la lengua materna" asume como problema de investigación la ausencia notoria de una evaluación formativa y auténtica de la lengua oral formal en nuestras aulas. Esto se debe, entre otras razones, a que el contexto escolar actual adolece de una tradición pedagógica y de una reflexión didáctica sobre la oralidad coherente con los desarrollos actuales de las ciencias del lenguaje.

Con el fin de realizar un primer acercamiento a esta problemática, la investigación, de carácter interpretativo, analizó las concepciones y las prácticas de enseñanza y evaluación de la lengua oral en seis colegios de educación secundaria de Bogotá D. C. Para ello se realizaron observaciones y grabaciones de clase de doce docentes y veinticuatro entrevistas semiestructuradas también dirigidas a docentes de la asignatura de lengua castellana. Se buscó identificar aspectos comunes respecto a la evaluación de la lengua oral, específicamente en lo que se refiere a ¿qué se evalúa?, ¿por qué y para qué?, ¿cómo se evalúa? y ¿con qué instrumentos?

Durante el proceso de recolección, análisis e interpretación del corpus se advierte la ausencia de planeación y preparación de estrategias que lleven a una reflexión permanente sobre los objetos de saber comprometidos en la comprensión y producción de la oralidad. Ello obedece a los modos como se conciben la enseñanza y el aprendizaje de la lengua oral.

El análisis de las observaciones de clases en distintos ciclos de escolaridad y los testimonios de los docentes revelan un desconocimiento de la evaluación de la oralidad como parte fundamental de la educación lingüística de los niños y jóvenes. Los resultados indican el predominio de una concepción normativa que valora aspectos formales de la lengua oral sustentados en su naturaleza acústica, efímera e informal.
Igualmente, se manifiesta la dificultad para evaluarla, tanto por la diversidad de componentes que se requieren como por la ausencia de referentes teóricos y didácticos que orienten la construcción de criterios y el diseño o adecuación de instrumentos.

Por otra parte, el análisis de las prácticas evaluativas de la oralidad manifiesta una ausencia de criterios para valorar las diferentes dimensiones presentes en la comprensión y producción de los géneros orales. Cuando se evalúa, la mayoría de docentes centra su mirada en aspectos formales de la lengua, como el orden de los enunciados, la pronunciación, la velocidad, el volumen o la entonación. Otros les dan importancia a aspectos proxémicos y quinésicos $y$, para la mayor parte, el dominio del tema o contenido es un criterio fundamental de valoración. Lo anterior se relaciona con la escasa formación en estas competencias en las propuestas curriculares que orientan los procesos de formación de docentes en lengua castellana.

El tratamiento dado a la evaluación de la oralidad en los diferentes registros de clase y en las entrevistas da cuenta, igualmente, de otros aspectos esenciales en su enseñanza y aprendizaje, tales como el papel de los agentes en dicha evaluación, los instrumentos utilizados y las consignas que orientan las actividades relacionadas con la comprensión y la producción oral de los estudiantes.

Sin duda, las implicaciones de los resultados anteriores plantean un desafío en torno a la necesidad de reconocer la importancia de las concepciones y prácticas del maestro y su incidencia en el desarrollo de la competencia discursiva. Esto nos lleva a establecer hipótesis acerca de la enseñanza y la evaluación de la oralidad y a proponer acciones tendientes a favorecer el desempeño oral de los estudiantes en diversos contextos de uso, tal como se propuso en la presente investigación. 
Raquel Pinilla Vásquez, María Elvira Rodríguez Luna, Yolima Gutiérrez Ríos, María Fernanda Torres, Martha Judith Camelo, Julia Esther Casjulia

Es importante señalar que en Colombia, en las últimas décadas, se evidencia un interés creciente por la didáctica de la oralidad como objeto de estudio y de aprendizaje. Tal reconocimiento se advierte en los Lineamientos curriculares (1998) y en los Estándares curriculares (2003) establecidos por el Ministerio de Educación Nacional de Colombia. En los Lineamientos se reconoce la importancia de la enseñanza y aprendizaje de la oralidad, y se contempla su desarrollo en el eje referido a la interacción y los procesos culturales implicados en la ética de la comunicación. Así mismo, en los Estándares se sostiene que la formación en lenguaje implica propiciar situaciones en las cuales tengan cabida los procesos de producción y comprensión oral involucrados en la actividad lingüística.

Así, por ejemplo, con los niños de preescolar se propone la realización de narraciones orales, recitación de poemas y actividades lúdicas, como trabalenguas y retahílas, entre otras. En los niveles de básica primaria y secundaria se plantea la ejecución de exposiciones orales, mesas redondas y representaciones teatrales. En los últimos grados se incluyen el debate y la asamblea. La mayoría de las veces, y solo en los niveles intermedios, estas actividades están precedidas por una fundamentación específicamente referida al tipo de discurso y sus características, por ejemplo la argumentación o la exposición, pero no sobre los requerimientos contextuales y discursivos de la interacción oral característicos de las situaciones comunicativas en las que tales actividades se realizan (Pinilla y Rodríguez, 2012).

Si bien las políticas curriculares anteriormente referidas plantean la necesidad de cualificar la comprensión y producción oral de los estudiantes y la importancia de una evaluación integral y formativa, no se profundiza sobre su naturaleza, sus modos de articulación, sus posibilidades de complejización, progresión y complementariedad con otras actividades del lenguaje. Tampoco brindan pautas a los docentes para ampliar y transformar postulados teó- ricos en representaciones didácticas comprensibles para los estudiantes. Como señala Rodríguez Luna, "los docentes creen que con proponer o permitir algunos debates o exposiciones en clase y corregir ciertos errores articulatorios, morfológicos, sintácticos o semánticos ya han cumplido con los objetivos de la comunicación oral" (2006, p. 61). Por tanto, la institucionalización de estos referentes de política curricular no garantiza una recontextualización o apropiación de nuevas prácticas (Gutiérrez, 2009).

\section{Presupuestos teóricos de la investigación}

Se asume que la oralidad como actividad del lenguaje debe ser objeto de reflexión sistemática y planeada en el aula y que sus aprendizajes deben ser evaluados. Se considera importante saber cómo insertar esta evaluación en los dispositivos pedagógicos y cómo construir criterios objetivos de evaluación como puntos de referencia. La reflexión sobre qué evaluar, cómo, cuándo y por qué hacerlo no solo favorece el desarrollo de competencias orales, sino que también pone en relación la oralidad y la escritura como actividades lingüísticas fundamentales en los procesos de enseñanza y aprendizaje.

Esta tarea investigativa remite a la necesidad de situar el problema de la enseñanza y la evaluación de la oralidad como objeto de conocimiento y reflexión. La contribución debe ser brindar herramientas conceptuales y prácticas que posibiliten la adecuada recontextualización de los avances logrados por las ciencias del lenguaje en cuanto al análisis de la lengua y el discurso oral, para ponerlos al servicio de la educación lingüística de los estudiantes.

Por lo anterior, es necesario acudir al estudio del conocimiento profesional del profesor, ${ }^{1} \mathrm{y}$ específica-

1 Cabe aclarar que el conocimiento profesional del profesor es un programa de investigación del cual se derivan numerosos estudios que dan cuenta de la importancia 
mente a sus concepciones, entendidas como "herramientas" para interpretar la realidad natural y social que pueden obstaculizar o, por el contrario, servir de vehículo para facilitar el proceso de construcción de conocimiento. Al respecto, Porlán y Rivero (1998) plantean que las concepciones actúan como un conjunto de ideas y comportamientos que poseen los profesores en relación con el conocimiento escolar y sus procesos de construcción. Estas ideas pueden manifestarse de forma explícita o tácita y tener un carácter epistemológico, didáctico-curricular, metodológico o experiencial. Esto sugiere el rol que cumplen las concepciones sobre un objeto de enseñanza y sus implicaciones en los aprendizajes de los estudiantes.

Los estudios realizados en este campo muestran una gran variabilidad en las concepciones de los docentes sobre la enseñanza de la lengua oral (Gutiérrez, 2011). Esta situación obedece a la relativa incongruencia entre el plano del discurso y el plano de la acción didáctica, es decir, el plano del hacer, situación propia de la fluctuación permanente de las concepciones y de su capacidad movilizadora y evolutiva. De ahí que en este estudio se plantee la importancia de analizar las concepciones mediante unos niveles organizados en gradientes de complejidad ascendente. ${ }^{2}$ Estos niveles actúan como referentes para determinar la progresión de las concepciones emergentes y señalan sus posibilidades de evolución y complejización:

- Nivel inicial: en el que predominan concepciones propias de una enseñanza y un aprendizaje

del análisis de las creencias, concepciones y modalidades de este conocimiento y su relación con la práctica pedagógica.

2 Este planteamiento se sitúa en la consideración del conocimiento profesional deseable que Porlán (1996) percibe como una posibilidad cambiante, representada mediante hipótesis de progresión o de referencia en cada nivel, las cuales dan cuenta de las posibles evoluciones de las concepciones de los profesores. academicistas y transmisionistas, correspondientes a un modelo didáctico tradicional. Suele ser de tendencia mayoritaria en los profesores de lengua castellana y literatura.

- Nivel intermedio: correspondiente a planteamientos o alternativas de innovación que intentan superar el modelo tradicional de la enseñanza y el aprendizaje. Los docentes asumen planteamientos diferentes para superar la tendencia mayoritaria propia del nivel inicial.

- Nivel de referencia: trata de superar las dificultades de los niveles anteriores y se aproxima al conocimiento profesional deseable.

Esta investigación asume la propuesta anterior planteada por Gutiérrez (2011), que entiende que la diversidad de concepciones y sus diferentes niveles de manifestación pueden ir de un nivel de simplicidad a un nivel de mayor complejidad o de referencia, lo cual significa que existen niveles de desarrollo intermedios. Para los fines del presente estudio se plantea analizar las concepciones sobre la evaluación de la oralidad mediante un gradiente que comprende tres niveles: uno inicial relacionado con concepciones de carácter estrictamente lingüístico, otro intermedio que incorpora elementos propios de concepciones de índole pragmática y comunicativa, y un nivel de referencia discursivo que relaciona las categorías anteriores.

La concepción lingüística, referida a la evaluación de la oralidad, se centra en aspectos formales de la lengua relacionados con el dominio de la voz. En consecuencia, privilegia los aspectos prosódicos de la expresión oral, tales como: la pronunciación, la entonación y proyección de la voz, la articulación, el ritmo, las pausas y los silencios. Además, se concentra en el análisis de la fluidez verbal en términos de la agilidad para seleccionar vocabulario y el número de palabras empleadas y su orden. 
Raquel Pinilla Vásquez, María Elvira Rodríguez Luna, Yolima Gutiérrez Ríos, María Fernanda Torres, Martha Judith Camelo, Julia Esther Casjulia

La concepción pragmática y comunicativa se sitúa en la construcción de sentido en el contexto en el que se desarrolla la comunicación oral. Para ello conviene tener en cuenta el uso real de la lengua oral; esto es, la adecuación de los distintos registros al contexto, el propósito comunicativo, la actitud del interlocutor, el control de la coherencia y cohesión de su discurso, lo cual implica reconocer la aparición de muletillas e incoherencias (fragmentaciones, ausencia de conectores, dispersiones) y sus posibilidades de autorregulación, si se atiende a qué se hace realmente cuando se habla y se escucha en determinadas situaciones comunicativas.

La concepción discursiva de la evaluación de la oralidad se instala en el dominio de los géneros orales y sus características particulares, en el reconocimiento de su informalidad o formalidad. Así pues, la conferencia, la exposición oral o la mesa redonda sugieren ciertos requerimientos lingüísticos, pragmáticos, comunicativos e incluso retóricos que distan en gran medida de una conversación familiar, por ejemplo. Se trata de una concepción que además valora la particularidad de las reglas sociales de cada contexto (cortesía lingüística, modalización, convenciones, etc.). Así mismo, reconoce el carácter multicanal de la oralidad, puesto que no se queda en lo puramente lingüístico, sino que lo conjuga con lo paraverbal, lo kinésico (gestos, movimientos, miradas) y lo proxémico (actitudes, posturas, hábitos culturales y, en general, la distancia con respecto al interlocutor).

Ahora bien, el enfoque teórico de esta investigación es la evaluación auténtica y formativa de los aprendizajes y toma como puntos de referencia, fundamentalmente, los desarrollos propuestos por Riestra (2008), Condemarín (2000), y Atorresi y Ravela (2009). Desde la perspectiva de estos autores, la evaluación auténtica constituye parte integral del proceso educativo, puesto que "busca mejorar la calidad y el proceso de aprendizaje y aumentar la probabilidad de que los estudiantes aprendan" (Condemarín,
2000, p. 17). Por su parte, la evaluación formativa implica un actividad orientada hacia el mejoramiento de los aprendizajes; su "principal objetivo [es] conducir los aprendizajes de los alumnos en el marco de una pedagogía diferenciada: llevar a todos los alumnos a dominar ciertas capacidades, a través de métodos y ritmos que respondan a sus necesidades particulares" (Condemarín, 2000, p. 39).

Una evaluación así orientada posibilita superar las prácticas sobre la oralidad contempladas en nuestras aulas, en su mayoría de carácter sumativo, ${ }^{3}$ y que se realizan al final de la tarea. Es decir, no apuntan al desarrollo de procesos de reflexión sobre la tarea ni a brindar elementos para superar las dificultades. Uno de los mayores aciertos de estos enfoques consiste en saber "dónde se encuentra el alumno respecto a un aprendizaje determinado, para saber mejor hasta dónde puede llegar" (Perrenoud, como se cita en Condemarín, 2000, p. 39).

La investigación asume también la propuesta de proyectos de evaluación formativa y auténtica planteada por Atorresi y Ravela (2009), quienes definen el proyecto como:

[...] la expresión de una conducta de anticipación que supone la capacidad de imaginarse algo que aún no es, pero que puede llegar a serlo en el tiempo futuro, mediante la construcción de una sucesión de actos y de acontecimientos. Es una representación del pensamiento en perspectiva, que permite proponer acciones tendientes a alcanzar una meta. (p. 1)

Por consiguiente, el proyecto debe partir de los intereses de los estudiantes e incluir situaciones problé-

3 Según Carlino (1999), esta evaluación "se utiliza cuando el propósito es evaluar la eficacia de los productos educativos" (p. 107). Para Castillo y Cabrerizo (2006), la evaluación sumativa tiene una función sancionadora en la medida en que "permite decidir el aprobado o no-aprobado de una asignatura; la promoción o no al siguiente curso; o la obtención o no de una determinada titulación" (p. 27). 
micas que requieren una serie de acciones planeadas y organizadas para su solución. En el caso de esta propuesta, las situaciones que se buscan resolver se refieren a las dificultades relacionadas con el uso de los diferentes géneros orales formales del discurso por parte de los estudiantes.

En cuanto a los instrumentos para la evaluación de la oralidad, ${ }^{4}$ se proponen, en primer lugar, las tablas de especificaciones sobre procesos y conceptos, explícitos y no explícitos, elaborados por el docente antes de la construcción del proyecto. En estas tablas se especifican los aprendizajes esperados. En segundo lugar, las matrices de coevaluación de los aprendizajes que contienen niveles de cumplimiento, criterios e indicadores referidos al uso del lenguaje oral, al género discursivo trabajado y a la interacción verbal. Mediante estas matrices se busca centrar la atención del estudiante en las competencias lingüística, sociolingüística, estratégica y discursiva. Igualmente, se propone una matriz para valorar el proyecto en la cual se incluyen criterios referidos a su organización, pertinencia, funcionalidad y compromiso con su desarrollo. Y en tercer lugar, a lo largo de su ejecución se utilizan listas de cotejo para autoevaluación y rejillas para valorar las construcciones orales.

\section{La evaluación de la oralidad en las aulas de educación secundaria de Bogotá}

Con el fin de dar respuesta las preguntas por el qué, cómo, con qué y por qué se realiza la evaluación de la oralidad, a continuación se presenta una lectura analítica e interpretativa del corpus representado en el decir (entrevistas) y en el hacer (observaciones y grabaciones de clase) de profesores de lengua castellana.

4 Se han adecuado instrumentos propuestos por Atorresi y Ravela (2009) para la evaluación de la escritura, incluyendo en ellos procesos e indicadores referidos a la oralidad y a los requerimientos de cada uno de los discursos orales de los proyectos adelantados por los docentes participantes en los talleres y seminarios.

\section{Concepción lingüística de la evaluación de la oralidad}

El corpus analizado muestra una tendencia predominante a la evaluación de elementos prosódicos que, según Contreras (2011, p. 273), hacen referencia a las particularidades sonoras de la lengua en el discurso, así como a la carga de expresividad y emocionalidad que el hablante agrega a la palabra. De esta forma, en el decir del maestro se encuentra que el aspecto valorado en mayor medida es el prosódico (73\%). La evaluación orientada por esta concepción también centra la mirada en la fluidez verbal, y en particular en el vocabulario, como aspectos importantes para ser evaluados. En los siguientes registros, se puede observar esta tendencia: ${ }^{5}$

Investigadora (I): Cuando desarrolla actividades relacionadas con la lengua oral, ¿qué aspectos evalúa y cómo los evalúa?

Docente (D) 1: El vocabulario, la entonación, el uso apropiado de las pausas, y se evalúa pues casi que individual, se hace una rejilla y se pide a los estudiantes que hagan autoevaluación, y la profesora recoge algunos aspectos que cada grupo presentó, cada estudiante, y se le hace la corrección. Por ejemplo, la entonación, o las pausas, o el tipo de vocabulario que están usando. Se evalúa de esa manera.

D5: La preparación del tema reflejada en la seguridad del exponente | dominio del tema | volumen de la voz | léxico o vocabulario | modulación-entonación | asertividad en lo que dice con respecto al tema | ritmo del discurso (monótono - pasivo - rápido - normal etc.) | convencimiento de lo que dice y de quienes escuchan | estilo formal manera como emite.

5 Los registros que se incorporan en el presente documento con el fin de ejemplificar, argumentar o ampliar las afirmaciones forman parte del corpus general de la investigación. Este fue obtenido durante el periodo 20102012, mediante grabaciones y observaciones de clases de doce docentes pertenecientes a seis instituciones de educación secundaria de Bogotá, quienes participaron de manera voluntaria en la investigación. 
Esta tendencia se manifiesta claramente en la evaluación de actividades orales como la exposición, la cual se evalúa de este modo:

D: Bueno / ahora vamos a seguir en una partecita que tiene que ver con la exposición que ellas hicieron / Manejaron un buen tono de voz / es decir / ustedes escuchaban lo que las chicas expresaban.

Estudiante: Sí.

D: ¿Sí? / ¿Ni muy alto ni muy bajo? / ¿Normal?

Por tanto, priman aspectos relacionados con el dominio de la voz sobre otros aspectos del discurso.

\section{Concepción pragmática y comunicativa de la evaluación de la oralidad}

Además de centrar la mirada en aspectos prosódicos, se consideran elementos relacionados con la intención, adecuación, cohesión y coherencia del discurso. Es así como priman criterios relacionados con la "claridad al hablar y el tono de la voz".

I: Cuando desarrolla actividades relacionadas con la lengua oral, ¿qué aspectos evalúa y cómo los evalúa?

D3: Utilización del lenguaje adecuado, actitudes que toma frente al grupo, el tono de la voz, la entonación y el conocimiento del tema.

Por ejemplo, en el siguiente registro de una exposición oral se evalúan la pronunciación y el ritmo y se tienen en cuenta el control de la coherencia y la cohesión del discurso:

8. D: ...sí bueno recordemos entonces los aspectos que se van a evaluar // claridad al hablar / es decir que se entienda lo que la persona está diciendo/ que haya un hilo conductor entre el tema que está desarrollando / y que se presenta al principio a través del título de la pregunta / y todo lo que la persona va explicando alrededor de ese tema / tono de voz que el tono de voz que utilice esa persona sea adecuado que todos lo escuchemos / muletillas vamos a mirar si esa persona utiliza muletillas o no / en el caso de que utilice muletillas le vamos a colocar ahí cuales son las muletillas que está utilizando /

Si bien los anteriores aspectos son importantes en la producción oral, no pueden evaluarse de manera descontextualizada, como tampoco deben constituirse en los únicos aspectos a ser tenidos en cuenta. De ahí la importancia de considerar la lengua oral en todas sus dimensiones, y de que su evaluación trascienda el aprendizaje sistemático de normas de pronunciación y aplicaciones repetitivas que determinan lo correcto e incorrecto de su uso, y no su adecuación al contexto en el cual se tengan en cuenta un tiempo, un lugar y unos actores (Atorresi, 2011).

De acuerdo con los registros anteriores, las concepciones del docente sobre la evaluación de la oralidad fluctúan entre el nivel inicial y el intermedio, por cuanto evalúa aspectos que corresponden a ambos. Sin embargo, su hacer y su decir expresan una mayor tendencia hacia la concepción lingüística. Cabe señalar que, en el decir, los docentes dan suma importancia a la evaluación del léxico:

I: Cuando desarrolla actividades relacionadas con la lengua oral, ¿qué aspectos evalúa y cómo los evalúa?

D7: Vocalización / manejo del grupo / agilidad mental / vocabulario y actitud hacia los demás.

No obstante, en la práctica una mínima representación de docentes (8\%) evalúa este aspecto. En el siguiente registro, se encuentra la coevaluación realizada por una estudiante a su compañero de clase quien acaba de realizar una exposición sobre mitos y leyendas. Los criterios de evaluación fueron entregados por la docente al iniciar la clase: "manejo del idioma, manejo del cuerpo y conocimiento".

D: ¿Cómo es el mito de las piedras de Tunja?

Estudiante (E) 1: Las piedras de Tunja se dice que fueron traídas por... 
E1: Pero si nos damos de cuenta que esas piedras quedaban en un lago y supuestamente los zipas traían esas piedras porque hicieron una promesa con el diablo, ¿sí? // también tienen zoomorforas / que son cosas como... [mira al docente] como... sí / como pinturas / antropomorforas que son como...

D: Esos se llaman códices.

E1: Bueno, códices...

D: Se le acabó la [...] // /a ver ¿ustedes qué le pueden ver a la niña? ¿cómo lo hizo?

E2. Bien.

D: [Da la palabra a una estudiante] Hable una

E3: Yo creo que / o sea, todo lo hizo bien el manejo del lenguaje la forma de moverse, la forma de expresarse, todo // lo que faltó fue darnos a conocer qué fue lo que sucedió más allá de (...) la leyenda.

D: Realmente [...]. Bueno ¿quién más? [Da la palabra. A ver ¿usted qué dice?

Como se muestra en el anterior registro, en el transcurso de la exposición el estudiante manifiesta falta de claridad en algunos términos utilizados en su discurso, tales como "zoomorforas" y "antropomorforas". Sin embargo, en la coevaluación realizada por un compañero no se reconoce esta dificultad, aspecto que el docente también pasa por alto.

A esta concepción pragmática y comunicativa se asocia una concepción de carácter didáctico relacionada con la organización y planeación de la enseñanza y el aprendizaje de un género oral. Aspectos tales como la inventio (selección del tema), la dispositio (organización a nivel macro, organización lógica del discurso) y la elocutio (organización a nivel micro, organización de la exposición de las ideas) deben integrarse tanto al plano de la enseñanza como al de la evaluación. A pesar de ello, en los registros analizados se encuentra una fuerte tendencia (85\%) a evaluar exclusivamente la consulta previa que el estudiante hace del tema y no la profundidad en su de- sarrollo. A continuación se cita un registro en el cual el docente presenta a los estudiantes algunos de los criterios, a nivel de la organización discursiva, para evaluar una exposición oral.

E: ¿Traer apuntes?

D: Ah bueno / traer de pronto unos apuntes / algo que me ayude / listo.

E: No quedarse callado $(())$.

D: Ah listo / eso tiene que ver con el manejo de la consulta / porque si yo / no vengo muy bien preparada / pues yo en un momento determinado me voy a quedar callada y no sé qué decir / y eso hace que el público se distraiga / bueno / que entre en otra situación.

En este caso, el docente tiene en cuenta dos aspectos: traer apuntes, lo que implicaría sintetizar o resumir el tema que es objeto de exposición, y manejo de la consulta, asumido como la profundización en la consulta. A pesar de tratarse de una clase en la cual se está trabajando la exposición oral como un género oral formal, a partir de ciertos rasgos de la concepción pragmático-comunicativa, el tratamiento que se les da a su preparación y evaluación sigue ligado a una concepción de nivel inicial, en la cual lo oral es asumido como algo espontáneo, ya que es el estudiante el único responsable de planear, organizar y presentar su exposición sin el debido acompañamiento previo del docente.

Situaciones similares ocurren frente a actividades en las que se solicita a los estudiantes dar cuenta del contenido de una obra literaria. La evaluación se limita a la "sustentación oral" del contenido, sin que medien procesos de reflexión conjunta sobre las particularidades de este género y sobre el proceso lector. Un ejemplo de ello se observa en el siguiente registro, cuando un docente de grado séptimo solicita a un grupo de estudiantes presentar el contenido de la obra literaria Juan Salvador Gaviota. 
Raquel Pinilla Vásquez, María Elvira Rodríguez Luna, Yolima Gutiérrez Ríos, María Fernanda Torres, Martha Judith Camelo, Julia Esther Casjulia

3. D: ¿Qué más? (10 s) / Cuenten la historia. / (7 s) Continúe contando la historia, usted /

[Uno de los estudiantes, con las manos en la espal$\mathrm{da}$, pegado al tablero vuelve a relatar algunos fragmentos de la historia. Posteriormente, una niña del grupo continúa con la narración con una postura similar a la de su compañero].

4. D: Continúe / ¿Qué más tiene que decir de lugares?

[Una estudiante continúa narrando la historia del libro].

5. D: ¿No más? / ¿No más que decir? (10 s)

6. E2: La enseñanza que deja el libro.

7. D: ¿La temática?/ Eso se llama temática. / (20 s)

\section{Concepción discursiva de la evaluación de la oralidad}

Es importante anotar que algunos profesores plantean a sus estudiantes realizar una evaluación integral del discurso oral, que contempla varios aspectos tales como: lo lingüístico, lo paralingüístico, lo pragmático y lo discursivo. Un ejemplo de ello es el siguiente:

2. D: [...] al finalizar la exposición / cada uno de ustedes va a tener un formatico / en el cual va a evaluar la exposición oral que hizo cada uno de los compañeros / y le va a dar una valoración cualitativa / diciendo las observaciones que se... / que es necesario mejorar / y las fortalezas de cada expositor / pero también le va a dar una valoración cuantitativa / es decir / numérica [...].

8. D: Sí, bueno, recordemos entonces los aspectos que se van a evaluar / claridad al hablar /es decir, que se entienda lo que la persona está diciendo /que haya un hilo conductor entre el tema que está desarrollando y que se presenta al principio / a través del título de la pregunta / y todo lo que la persona va explicando alrededor de ese tema / tono de voz / que el tono de voz que utilice esa persona sea adecuado / que todos lo escuchemos / multillas / vamos a mirar si esa persona utiliza muletillas o no / en el caso de que utilice muletillas, le vamos a colocar ahí cuáles son las muletillas que está utilizando / manejo de organizadores gráficos / recordemos que vamos a mirar allí si la persona utiliza adecuadamente los organizadores gráficos que construyó / para apoyar lo que está diciendo en forma verbal / postura corporal / en la postura corporal vamos a mirar lo que hablábamos ayer / si esa persona en su postura corporal es adecuada para el momento de la exposición / el dominio del tema / si durante toda la exposición / yo puedo decir al final / que esa persona demostró que dominaba el tema que había sido previamente estudiado/ y que realmente el tema me quedó a mí claro / interacción con el auditorio / en la interacción con el auditorio vamos a mirar si la persona / por ejemplo / establece una comunicación conmigo / bien sea desde la mirada / desde las preguntas[...].

Nótese que el docente, antes de iniciar las exposiciones orales, plantea cinco criterios: claridad, tono de voz, muletillas, manejo de organizadores gráficos, interacción con el auditorio, que abarcan tanto aspectos lingüístico-discursivos, como paralingüísticos y formales.

En el siguiente registro, igualmente encontramos elementos de la concepción discursiva de la oralidad:

D: Ya habíamos visto cómo se hace una exposición I y eso requiere de unos pasos para que la exposición sea lo mejor posible / dentro de esos pasos lo primero que hay que hacer es identificar el tema del cual vamos a tratar / el tema / exposición / otro es determinar cuál es la estructura más adecuada para poder hacer la exposición / lo otro, consultar la información sobre el tema en el libro / en Internet / en revistas / estar con información completa sobre el tema / otro / ordenar la información seleccionada por temas y / subtemas / luego / teniendo ya esto, redactar la información empleando un lenguaje claro / preciso / y adaptado al nivel de conocimientos del receptor / $((\ldots))$ su preparación del tema, de tal manera que sus compañeros entiendan qué información quiere usted dar a conocer / luego, elaborar y preparar el material de apoyo / que vamos a utilizar para ayudarnos a dicha exposición y también 
recuerden que la exposición la pueden hacer de varias formas / una de ellas es la actuación / que vimos cómo debe hacerse / lo otro es el discurso que se emite / si sí estamos llegando a ese público receptor / o no estamos llegando a él / y la última es la interacción con el auditorio / a medida que ustedes van haciendo la exposición / se dan cuenta si / el público está animado / si le está poniendo cuidado / o el público está aburrido / si el público y no le está escuchando / ¿qué debemos hacer? / [...] además de toda la teoría del tema / cómo debe estar la presentación del expositor... / debemos venir de acuerdo a la exposición que tengamos / vamos a vestirnos...

Como se observa, el docente centra su atención, en primera instancia y de manera reiterativa, en la preparación de la exposición, en la necesidad de optimizar la interacción con el auditorio, mantener su atención y demostrar dominio del tema. Además, enfatiza la presentación del expositor y hace referencia a aspectos semánticos y pragmalingüísticos relacionados con la organización del discurso, la claridad, la precisión y la adecuación al contexto y al nivel de conocimientos del receptor.

De esta forma, se manifiesta en el hacer del profesor una concepción que fluctúa entre lo lingüístico, lo pragmático y lo discursivo, pese a que omite aspectos relacionados con lo paralingüístico, proxémico y kinésico, y otros propios del discurso oral, que contribuyen a dotar de mayor sentido el evento comunicativo.

No obstante, algunos profesores (33\%) en sus evaluaciones omiten algunos de los aspectos anteriores, aunque le dan relevancia a la proxemia, entendida esta como la proximidad entre los interlocutores, en la cual se construye la actuación performativa del acto oral, en tanto le interesa la relación espacial (contexto), esto es, la lejanía o cercanía de los interlocutores (Contreras, 2011, p. 279). De esta manera, el manejo de los objetos que se encuentran inmersos en el acto comunicativo oral, tales como apretar las manos o dar golpes en la espada a alguien, son ac- ciones que también cobran sentido en el momento de la interlocución oral. La distancia de un hablante con su interlocutor lleva a crear un ambiente y una predisposición en el hablante que, sin lugar a dudas, va a incidir en el significado semántico del discurso. El siguiente registro inicia con una evaluación de aspectos proxémicos, como "mirar al público" y mantener una postura en dirección al auditorio para asegurar la interacción.

D: [...] / bueno / y en cuanto al manejo de las niñas / mirando al público / ¿cómo lo vieron? / ¿Estuvieron ellas de frente / o sea / mantuvieron la comunicación visual con ustedes?

E: Sí.

D: Sí / exacto / Mantener la comunicación visual es importante / porque en la exposición no tendría sentido que yo / simplemente diese la espalda / ¿ cierto?/ Diese la media vuelta / y todo el curso que me vieran esta parte [mostrando la espalda] / porque ¿cuál es la parte que comunica? / Los ojos / ¿cierto? La expresión... / Siéntate bien que te puedes caer... / La expresión de la cara en los ojos / específicamente es lo que mantiene la comunicación entre la audiencia y el que expone / ¿cierto?//

En otros casos, como en la representación de las obras de teatro, los docentes centran su atención en la evaluación de elementos kinésicos, específicamente en los movimientos del cuerpo, en los gestos, la mirada, la sonrisa, los movimientos de la cabeza, los desplazamientos etc. Aun así, desde el concepto de expresión corporal, este tipo de trabajos teatrales no son orientados por los docentes sino que se trata de acciones que los estudiantes preparan de manera intuitiva, que sin embargo son evaluadas. Un ejemplo de este caso es la siguiente autoevaluación realizada por un grupo de estudiantes de grado décimo después de realizar la representación teatral de unas obras literarias. Entre los criterios que propuso la docente para evaluar el nivel proxémico y kinésico se encuentran: la relación con el público, el manejo 
del escenario, los movimientos adecuados y la expresión corporal:

E1: Y en cuanto a la expresión el trabajo fue bien expresado eh | pues las luchas y todo eso que se maneja estaba en la puesta en escena /

48. E2: Bueno / pues la expresión corporal pues / nosotras tratamos de manejar al máximo o sea como // movimientos que concordaran o sea con lo que estábamos diciendo / o con lo que decía la historia /

Como se puede corroborar en el anterior registro, la docente expone los criterios de evaluación y posteriormente solicita a los estudiantes que pasen y realicen su autoevaluación. Aunque los criterios abarcan una visión amplia de la evaluación de lo oral, no poseen indicadores específicos para cada uno, lo cual permitiría a los estudiantes tener mayores elementos de juicio para su autoevaluación. Además, estos criterios fueron presentados a los estudiantes solo al final del trabajo y su construcción estuvo exclusivamente a cargo del docente. Lo más preocupante es que evalúan aspectos que no fueron trabajados previamente, ya que en este tipo de actividades el docente se limita a plantear el tema y a establecer como actividad la representación de obras teatrales.

Es así como, a pesar de involucrar evaluaciones en las cuales se evidencian algunos rasgos de una concepción discursiva, sigue primando una concepción lingüística de las actividades orales.

\section{Modalidad de la evaluación}

En cuanto a las modalidades de evaluación de la lengua oral presentes en el aula, se identifica una fuerte tendencia $(83 \%)$ a la realización de aquellas con carácter certificativo desarrolladas al final de la actividad oral propuesta. Generalmente, estas evaluaciones finales se centran en la valoración del producto y dejan de lado el proceso de diseño, elaboración y adecuación del discurso.
En su gran mayoría, se trata de evaluaciones de carácter certificativo mediante las cuales se pretende verificar el nivel de aprendizaje del estudiante y el grado de consecución de los objetivos propuestos en la asignatura. Se tiende a relacionar esta evaluación con la sumativa y final. Al respecto, Castillo y Cabrerizo (2006) indican que, "aunque no sea necesariamente sumativa, la evaluación final suele identificarse con ella en la medida [en] que enjuicia o valora procesos finalizados haciendo acopio de los datos seleccionados en los anteriores momentos de la evaluación" (p. 26).

En los registros analizados se encontró esta modalidad de evaluación evidenciada en los comentarios orales realizados por el docente después de la presentación de una actividad oral. Un ejemplo de ello es la siguiente evaluación que realiza un docente a sus estudiantes de grado sexto después de la presentación de una exposición oral.

D: La exposición se debe preparar con anterioridad / debieron haber leído e informarse más / y en una exposición debe estar claro cuál es el tema / cuál es el objetivo / qué quieren de dicho tema / haber preparado unas carteleras [...]

Como se puede observar, el docente manifiesta a sus estudiantes los aspectos en los que fallaron a nivel de la planeación - "haber leído e informarse más"- y a nivel formal - "haber preparado unas carteleras"-. Así mismo, el docente les hace un llamado de atención en relación con la falta de preparación previa para la actividad, lo cual deja entrever que esta fue asignada por el docente y diseñada por los estudiantes sin ningún tipo de orientación. Esto pone en evidencia una concepción de la enseñanza y de la evaluación de lo oral como actividad espontánea en la cual el docente evalúa un resultado sin tener en cuenta el proceso previamente desarrollado por los estudiantes.

Condemarín (2000, p. 11) considera este tipo de evaluaciones como tradicionales ya que se caracte- 
rizan por prácticas en las que el maestro, después de enseñar, administra una prueba o interroga a los alumnos oralmente con el fin de recuperar la información planteada en el proceso de enseñanza. Son evaluaciones generalmente certificativas y no constituyen una instancia para el mejoramiento de la calidad de los aprendizajes. Así mismo, dejan de lado los contextos en los cuales se desarrollan las actividades pedagógicas. Son unidireccionales, ya que los resultados son utilizados solamente por el docente. Y son excluyentes, en cuanto generan jerarquías de excelencia.

Estas evaluaciones finales son utilizadas por los docentes para expresar algunos "consejos" o "sugerencias" que los estudiantes deben tener en cuenta para sus próximas actividades discursivas orales. En escasas ocasiones se constituyen en aprendizajes significativos para los estudiantes, ya que son generalizaciones que se hacen para el grupo de manera oral, sin el debido seguimiento mediante algún instrumento escrito.

\section{Agentes de la evaluación}

En cuanto a los agentes que desarrollan el ejercicio evaluativo, se encuentra que en un alto porcentaje (63\%) predomina la heteroevaluación ${ }^{6}$. Este tipo de evaluación, realizada por el docente al finalizar una actividad oral, se hace a partir de comentarios orales sin criterios previamente establecidos. Es así como la evaluación deja de ser una actividad integrada al proceso de enseñanza, a partir de la cual los estudiantes puedan planificar y desarrollar los objetivos

6 La heteroevaluación es "la modalidad en la cual los evaluadores y los evaluados no son las mismas personas quienes toman la decisión de planificar y ejecutar la evaluación. Es la evaluación más común (a la que estamos habituados) ya que generalmente es el docente quien se encarga de evaluar el desempeño de los estudiantes" (Atorresi, 2011, p. 46). planteados en la clase, y se convierte en un elemento netamente sancionatorio.

Otro agente evaluador presente en las prácticas observadas es el estudiante. Esta modalidad denominada coevaluación representa un porcentaje bastante significativo (27\%) y se caracteriza por que los evaluadores y los evaluados intercambian su papel alternativamente. Para Castillo y Cabrerizo (2006, p. 91), la coevaluación permite, por un lado, tomar conciencia respecto de los avances y problemas individuales y grupales; $y$, por otro, desarrollar una serie de desempeños sociales como convivencia, solidaridad, sociabilidad, respeto mutuo, entre otros. En los registros analizados se encuentra que la coevaluación, al igual que la heteroevaluación, se realiza al finalizar la actividad discursiva oral propuesta, y que es el docente quien asigna los criterios y los puntajes que se utilizarán para la evaluación.

Estas modalidades de evaluación, aunque son motivadoras porque permiten que los estudiantes asuman mayor protagonismo en su proceso de aprendizaje, también implican un trabajo de mayor responsabilidad por parte del docente. Antes de iniciar la actividad, se requiere revisar con los estudiantes las características e implicaciones de los criterios de evaluación, así como la negociación de estos, para lograr que la evaluación se constituya en un proceso formativo.

Al respecto, Álvarez (2001) considera que la negociación de los criterios de la evaluación permite una práctica democrática y participativa que le ayuda al docente a "llegar a tiempo para evitar el fracaso escolar". En consecuencia, no basta con establecer criterios de evaluación, sino que estos deben ser consensuados con los estudiantes que serán sujetos de la evaluación y deben servir de guía en la preparación de la actividad discursiva oral. Así mismo, es importante que estos criterios cuenten con una lista de especificaciones para que cuando los estudiantes 
vayan a realizar el papel de evaluadores tengan mayores herramientas al momento de hacer sus apreciaciones y se evite caer en comentarios generales sin mayor análisis.

Finalmente, en menor proporción se evidenció la presencia de autoevaluación (10\%). En esta modalidad de evaluación el estudiante es considerado como un elemento activo y responsable dentro del proceso evaluador. Para Álvarez (2001), la autoevaluación no es ni puede ser apéndice de la enseñanza. Es parte de la enseñanza y del aprendizaje, ya que, en la vida académica, quien sabe realmente si aprendió o no y por qué es el sujeto que aprende. Por ello, la base fundamental para la autoevaluación son el análisis y la reflexión que hace el sujeto sobre su proceso, y sobre lo que ocurre durante este, para tomar las decisiones pertinentes. Esto implica un cambio radical en la concepción de evaluación, en sus procesos y en la utilización de instrumentos para evaluar.

En los registros analizados, se encontró que esta modalidad de evaluación se propone en el aula como ejercicio de reflexión personal, especialmente sobre aspectos procedimentales implicados en la actividad. Al igual que la heteroevaluación y la coevaluación, se realiza al finalizar la actividad y es el docente quien especifica los criterios. A continuación se muestra la consigna dada por la docente para que sus alumnos se autoevalúen después de la presentación de las obras de teatro sobre los relatos de la mitología griega. Con este ejercicio se buscaba evaluar la comprensión de lectura de mitos y leyendas clásicos e identificar las características de la literatura de la época.

[El docente escribe los aspectos a evaluar en el tablero: vestuario ("parafernalia"), manejo de la voz, puesta en escena, expresión corporal, ayudas audiovisuales].

D: Entonces, ¿qué vamos a hacer ahora? // voy a nombrar a cada grupo // y van a pasar y hablan de cada uno de los aspectos // toman su nota / dan su nota // y / los demás grupos también les pueden dar una / si están de acuerdo con esa nota o no están de acuerdo, ¿sí?, o si les pareció la obra muy buena entonces dicen: "no, ellos se merecen más nota, sacan tanto" / miramos también la apreciación del público, algo muy importante [la docente escribe este aspecto en el tablero].

D: Parafernalia es el vestuario que utilizaron.

D: Pasen ustedes de primeras // primero nos van a comentar cómo se sintieron trabajando / qué dificultades tuvieron y vamos a mirar cada uno de los aspectos // ¿cuántos son del grupo?

D: Analizan y miran cómo fue cada uno de los aspectos.

E: Pues / la para, parafernalia a mí me pareció muy chévere porque todos llevamos el vestuario que se utilizaba en esa época / y pues representamos bien los personajes que / nos correspondieron.

La implicación de los estudiantes en el proceso de evaluación de la oralidad es un avance importante, pese a que los criterios siempre son propuestos por el profesor y adolecen de claridad conceptual, como en el caso del anterior registro.

Como se ha evidenciado hasta el momento, para la evaluación de la lengua oral predominan los ejercicios que en su mayor parte realiza el docente al finalizar la actividad oral propuesta. Esta se desarrolla a partir de comentarios orales, en algunas ocasiones direccionados por criterios de evaluación que el docente expone al momento de aplicar la evaluación y, en general, no son conocidos con anterioridad por el grupo de estudiantes.

\section{Instrumentos de evaluación}

Se consideran instrumentos de evaluación aquellas herramientas externas que actúan como mediadores del proceso de enseñanza y aprendizaje. Generalmente se trata de tablas o matrices diseñadas por el 
docente y aplicadas al grupo de estudiantes que son objeto de evaluación. Estos instrumentos son elaborados a partir de criterios que especifican los aspectos lingüístico-discursivos y paralingüísticos que son objeto de evaluación. En principio, los criterios son establecidos por el docente para la clase, aunque el objetivo es lograr que los estudiantes, con el tiempo, se involucren en su diseño y aplicación.

En el caso de la oralidad, son escasos el diseño y aplicación de instrumentos de evaluación diferentes al comentario oral. En los registros analizados se encontró que en un bajo porcentaje (25\%) se hace uso de rejillas de evaluación. Estas consisten en un listado de criterios que el docente propone al curso al momento de iniciar el proceso evaluativo. Generalmente son matrices de dos entradas. Una de ellas está constituida por criterios generales de evaluación y la otra, por la valoración afirmativa o negativa de la actividad. En algunos casos se encuentran exclusivamente los criterios y una nota numérica que totaliza la evaluación.

Uno de los casos que muestra claramente este tipo de instrumentos es el siguiente registro de una clase de lengua castellana de grado décimo, en la cual la docente propone una rejilla para evaluar un debate.

379 P: Ee (aaaa) / dos / hay uno o dos que vamos a hacer como de jurados // del desarrollo del debate / y me van a llenar la siguiente rejilla /// me van a llenar la siguiente rejilla / recuerden que la rejilla /es $\rightarrow$ / a ver / silencio / la rejilla es de doble entrada, donde están por un lado los estudiantes que van a, los estudiantes que van a opinar / y por otro / los criterios que vamos a mirar // ¿qué criterios vamos a mirar aquí en esta ocasión? / participación / oiga / porque ustedes tienen que tenerla en cuenta / participación ordenada y oportuna / ordenada / ... // calidad // calidad de las participaciones / vamos a tener en cuenta la calidad / la calidad, nos vamos a referir sobre todo a cómo se dirigen al otro / al contrincante / sin ofenderlo, sin usar palabras despectivas // ... brevedad / o sea, precisar lo que quiero decir / no empezar con ejemplos extensos ... relevancia / o sea, que sea importante lo que dice / y pertinencia / que sea lo que tiene que decirse aquí en el en el tema / sin salirse del tema / de aquí hay un total de / esa participación / aquí lo vamos a dar // un punto a esto / a la participación ordenada y oportuna.

En el anterior instrumento, la docente propone una rejilla que consta de la lista de estudiantes de la clase y seis criterios que hacen referencia a las máximas conversacionales propuestas por Grice: participación, calidad, brevedad, relevancia y pertinencia. ${ }^{7}$ Cada uno de los aspectos es evaluado a partir de puntos que al final son sumados para obtener el resultado final. Esta rejilla se caracteriza por enunciar los criterios pero no los especifica ni los pondera, de tal forma que el evaluador pueda decir en qué nivel el estudiante alcanzó el criterio evaluado. Por otra parte, se trata de una rejilla que escasamente evalúa el nivel conversacional y deja de lado otros aspectos importantes de la oralidad.

A pesar de encontrar un incipiente desarrollo de instrumentos de evaluación sobre lo oral, es importante destacar que también algunos docentes $(8 \%)$ dan muestra de un avance significativo en sus concepciones de la evaluación de lo oral, aportando rejillas de evaluación en las cuales se tienen en cuenta aspectos lingüísticos y paralingüísticos. Este aspecto fue

7 Paul Grice (1975) concibe los intercambios orales como esfuerzos cooperativos que se basan en el siguiente principio general: "haz que tu contribución a la conversación sea la necesaria en el momento en que se da, con base en el propósito o dirección captado dentro del intercambio de conversación en el que estás comprometido. Esto podríamos llamarlo principio cooperativo. Partiendo de que un principio general tal como este es aceptado, podríamos quizás distinguir cuatro categorías bajo las cuales cabrán ciertas máximas y submáximas más específicas, y que por lo general tendrán resultados en relación con el principio cooperativo. Siguiendo a Kant, yo llamo a esas categorías cantidad, cualidad, relación y modo" (pp. 106-107). Traducido por Julio César Mejía en Lenguaje y Sociedad (1973). 
Raquel Pinilla Vásquez, María Elvira Rodríguez Luna, Yolima Gutiérrez Ríos, María Fernanda Torres, Martha Judith Camelo, Julia Esther Casjulia

igualmente señalado por Cárdenas y Torres (2011) en su investigación sobre la evaluación en el aula.

Como se expuso en el análisis de los anteriores registros, se puede afirmar que las rejillas son el único instrumento de evaluación utilizado por los docentes, ya que en ninguna de las grabaciones y entrevistas realizadas se observó el uso o diseño de instrumentos tales como listas de cotejo, pautas de evaluación, tablas de especificaciones o matrices ponderadas.

De esta forma, se considera que el escaso uso de instrumentos de evaluación para las actividades orales de la lengua es una muestra más de la insuficiente presencia de la enseñanza de lo oral en las aulas de clase. Así mismo, deja a la luz una concepción de lo oral como algo espontáneo, que quizás no requiere de un seguimiento tan riguroso como sí lo ameritan otras actividades del lenguaje, como la escritura y la lectura.

\section{Las consignas en la evaluación de lo oral}

En la evaluación de lo oral es importante tener en cuenta la consigna o instrucción que el docente formula, por cuanto esta, como texto, orienta y dirige las acciones que adelantará el estudiante para la preparación y presentación de una actividad oral. Del mismo modo, la instrucción define los aspectos que se tendrán en cuenta para valorarla. Sin embargo, en la mayoría de los casos, en las consignas planteadas están ausentes los criterios para evaluar la actividad discursiva oral del estudiante. Así lo muestra el siguiente registro de una docente: "Vamos a leer por grupos los mitos griegos y vamos a organizar y a presentar una obra de teatro".

En este caso, la instrucción se encamina hacia la verificación de la lectura de los mitos griegos. Tal como señala Camelo (2012) en su investigación, consignas como esta no favorecen la realización de una actividad oral formal en un contexto auténtico.
Con ellas se busca que el estudiante replique un conocimiento sin atender a criterios específicos, sin revisar previamente modelos de los diferentes géneros discursivos orales y sin contar con las pautas claras para su ejecución. La instrucción carece de autenticidad porque no favorece la proyección de los aprendizajes en la vida cotidiana del estudiante, es decir, la producción que se propone, en estos casos, no cobra ningún sentido o significado real para el estudiantado. A este respecto, Atorresi (2011) plantea que "una tarea es auténtica cuando propone actuar un rol que saca al estudiante de las tareas que realizaría si solo fuera estudiante" (p. 3).

Cabe destacar que para el desarrollo de la oralidad formal es necesario reconocer la definición y el concepto de cada uno de los géneros discursivos, unidos al estudio y revisión de distintos modelos para que el estudiante se apropie con mayor facilidad de estos. En la escuela suele trabajarse, especialmente en los cursos avanzados, la conceptualización de diferentes géneros orales, pero rara vez se acude a ejemplos en situaciones reales. Así, asumiendo la relevancia de la oralidad en la sociedad es conveniente "intervenir en el desarrollo de la competencia oral de los ciudadanos lo cual significa facilitar a los estudiantes el acceso a los usos formales" (Abascal, 1993, p. 160). Por lo tanto, el planteamiento de las consignas en contextos auténticos favorece el acercamiento, la realización y la evaluación de textos orales en el aula de manera auténtica y formativa.

\section{Discusión de los resultados}

El análisis de las concepciones de los docentes de lengua castellana con respecto a la enseñanza y la evaluación de la oralidad manifiesta una fluctuación permanente entre una concepción lingüística, una pragmática y comunicativa, y una discursiva, porque se tienen en cuenta algunos aspectos derivados de cada una de ellas. Es significativo que haya una tendencia mayoritaria hacia la pragmática comunicativa. 
Esto puede interpretarse como una conciencia por involucrar ciertas dimensiones de la oralidad que contribuyen al desarrollo de la competencia discursiva oral de los estudiantes.

En cuanto a la poca presencia de aspectos relacionados con la concepción discursiva, esta indica que hay una ausencia de formación al respecto, tal como se ha señalado en este documento. En efecto, evaluar la producción oral desde una concepción discursiva implica reconocer el nivel de desarrollo de la competencia discursiva oral del estudiante. A su vez, requiere el diseño de herramientas heurísticas que hagan posible la reflexión lingüístico-discursiva, a fin de que se garantice un dominio sobre lo que se dice y la manera como se dice (coherencia, adecuación, cohesión, cooperación y cortesía). Para ello se requiere incorporar diversas tipologías discursivas (narrativas, explicativas, argumentativas, conversacionales); acudir a estrategias de comprensión como la anticipación y la síntesis, a la armonía en el uso de recursos verbales y no verbales, al uso coherente de aperturas, desarrollos y cierres, y, en consecuencia, al control de las emociones y miedos propios de la oralidad pública (Gutiérrez, 2011).

En general, las actividades planteadas por el docente no se orientan por criterios explícitos para su evaluación, debido a que no cuentan con los fundamentos conceptuales ni las herramientas metodológicas para adelantar de manera coherente y pertinente este proceso. Evidentemente, y como lo señala García-Debanc (1999), evaluar lo oral es mucho más complejo que evaluar lo escrito debido a su naturaleza fugaz, cambiante y de difícil registro, entre otros aspectos, pero no por ello es imposible. Esta situación se presenta de manera similar en otros países, como lo señalan las investigadoras españolas Palou et al. (2005): "a la hora de concretar los ítems para la evaluación de la lengua oral, los docentes se limitan con frecuencia a aspectos generales del tipo 'participa' 'no participa’ o 'desarrolla la imaginación”' (p. 40).
Este problema se explica tanto por razones de orden teórico, relacionadas con la diversidad de componentes que se requieren para el análisis del discurso oral, como también por las condiciones empíricas de nuestras aulas, en las que muchas veces se carece de los materiales necesarios y de los dispositivos tecnológicos. Además, hay que considerar el gran número de estudiantes por aula a quienes cada docente debe orientar y evaluar, individual y colectivamente.

Por otra parte, las propuestas de instrumentos de evaluación de lo oral que existen actualmente son escasas. En general se limitan a listas de cotejo o rejillas centradas fundamentalmente en aspectos que acompañan a la actividad discursiva oral (no verbales y paralingüísticos), pero que no constituyen su esencia. El hecho de no contar con los instrumentos adecuados para evaluar la oralidad refleja incomprensiones de la especificidad de esta actividad del lenguaje y este hecho dificulta la evaluación de los aprendizajes. En efecto, al realizar una revisión de las investigaciones acerca de la evaluación de lo oral, se encuentra que son escasos los trabajos que proponen instrumentos para su valoración.

En relación con la escucha, las entrevistas y observaciones realizadas muestran una ausencia notoria de su enseñanza. Así lo reconocen los profesores, quienes argumentan que no cuenten con herramientas que les permitan una cabal comprensión de la complejidad de esta actividad, tal como se advierte en la siguiente intervención de una docente participante en la investigación: "[...] la escucha es uno de los problemas de la escuela; a pesar de estar inmersos en un universo de sonidos, no se escuchan ni escuchan a las demás personas, no hay eco de las palabras, comprensión". La escucha, según Núñez Delgado:

[...] implica una atención despierta, activa, que formula preguntas y sugiere respuestas, que se anticipa a la acción futura que tal vez va a desplegar la audición. Escuchar engloba todo el proceso del pensamiento $[\ldots]$. Escuchar es comprender un mensaje 
Raquel Pinilla Vásquez, María Elvira Rodríguez Luna, Yolima Gutiérrez Ríos,

María Fernanda Torres, Martha Judith Camelo, Julia Esther Casjulia

por medio de la puesta en marcha de un complejo proceso cognitivo de construcción de significados y de interpretación de un discurso pronunciado oralmente. (2003, pp. 171-172)

Teniendo en cuenta este planteamiento, la enseñanza y el aprendizaje de la escucha deben contar con criterios e instrumentos que permitan su evaluación, de manera tal que les sea de utilidad a los estudiantes para ser conscientes de sus fortalezas o debilidades, y que puedan superarlas o afianzarlas. Se trata, por lo tanto, de sentar las bases de un nuevo tipo de evaluación que se aparte de las tradicionales funciones de clasificación, certificación y sanción, y se constituya en herramienta para la transformación de las prácticas de enseñanza y aprendizaje de la lengua oral.

\section{Conclusiones}

Si bien la enseñanza y evaluación de la oralidad en la escuela es un asunto que compete a todo el profesorado, este estudio sobre las concepciones de los profesores de lengua castellana en torno a la enseñanza y evaluación de la oralidad da cuenta de la necesidad de prestar atención a la formación y actualización de los docentes de esta área del conocimiento. Ellos sonlos llamados, en primer lugar, a aportar en el desarrollo de la competencia discursiva oral de los estudiantes.

Desde esta perspectiva, la investigación contribuye a la cualificación de las prácticas educativas de un grupo de docentes de lenguaje, fortaleciendo su conocimiento profesional y su reflexión desde la coherencia entre su decir y hacer. De esta manera, es posible lograr las transformaciones necesarias que permitan cualificar la enseñanza de la oralidad en el aula.

Los resultados que se obtienen permiten identificar las competencias orales que requieren de cualificación y, a su vez, aportan herramientas conceptuales y prácticas que posibilitan la adecuada recontextualización de los avances logrados por las ciencias del lenguaje y de la pedagogía, en cuanto a la evaluación de la lengua materna, y particularmente de la comprensión del discurso oral.

\section{Reconocimientos}

Este artículo es resultado de la investigación "Evaluar lo oral para cualificar las prácticas de enseñanza-aprendizaje de la lengua materna", financiada por el Centro de Investigaciones y Desarrollo Científico de la Universidad Distrital Francisco José de Caldas, contrato n. ${ }^{\circ} 63$, código 571.

\section{Referencias}

Abascal, D. (1993). La lengua oral en la enseñanza secundaria. En El enfoque comunicativo de la enseñanza de la lengua (pp. 159-179). Barcelona: Paidós.

Álvarez, J. (2001). Evaluar para conocer, examinar para excluir. Madrid: Morata.

Atorresi, A. (2009). Segundo Estudio Regional Comparativo y Explicativo (Serce). Aportes para la enseñanza de la lectura. Santiago de Chile: Oficina Regional de Educación Unesco para América Latina y el Caribe y Laboratorio Latinoamericano de Evaluación de la Calidad de la Educación (Llece).

Atorresi, A. (2010). Construcción y evaluación de consignas de resolución escrita. Buenos Aires: Flacso Argentina.

Atorresi, A. (2011). Seminario de evaluación. Bogotá: Maestría en Pedagogía de la Lengua Materna, Universidad Distrital Francisco José de Caldas.

Atorresi, A. y Ravela, P. (2009). Los proyectos de evaluación formativa y auténtica. Montevideo: Instituto de Investigaciones en Evaluación, Universidad Católica del Uruguay. 
Bojacá, B., Morales, R. y Bustamante, B. (2000). Relación entre concepciones sobre el lenguaje y la lengua escrita. Revista Enunciación (Universidad Distrital Francisco José de Caldas, Bogotá) 4-5, 109-118.

Camelo, M. (2012). La consigna en la producción y evaluación de la oralidad y la escritura. Trabajo de grado no publicado, Maestría en Pedagogía de la Lengua Materna, Universidad Distrital Francisco José de Caldas, Bogotá, Colombia.

Cárdenas J., Torres M. F., Camelo, M. Gutiérrez, Y., Pinilla, R y Rodríguez M. E. et al. (2011, enero-junio). Hallazgos iniciales sobre evaluación de la lengua oral en el aula. Revista Enunciación (Universidad Distrital Francisco José de Caldas, Bogotá), 16 (1), 70-84.

Cárdenas, E. J. y Torres, M. F. (2011). Análisis de las prácticas evaluativas en lectura, escritura $y$ oralidad. Un camino hacia la reflexión sobre la evaluación en el aula. Trabajo de grado no publicado, Maestría en Pedagogía de la Lengua Materna, Universidad Distrital Francisco José de Caldas, Bogotá.

Carlino, F. (1999). La evaluación educacional. Buenos Aires: Aique.

Castillo, S. y Cabrerizo, J. (2006). Evaluación educativa y promoción escolar. Madrid: Pearson.

Condemarín, M. (2000). Evaluación auténtica de los aprendizajes: un medio para mejorar las competencias en lenguaje y comunicación. Santiago de Chile: Andrés Bello.

Condemarín, M. y Medina, A. (2002). Evaluación auténtica del lenguaje y la comunicación. Madrid: CEPE.
Contreras, I. (2011). Fundamentos teóricos para el estudio de la oralidad. En R. Pinilla y Y. Gutiérrez (comps.), La oralidad en contextos diversos: aportes investigativos para su discusión y comprensión (pp. 261-282). Bogotá: Universidad Distrital Francisco José de Caldas.

Escamilla, A. y Llanos, E. (1995). La evaluación del aprendizaje y de la enseñanza en el aula. Zaragoza: Luis Vives.

García-Debanc, C. (2010). Evaluar lo oral (R. Pinilla y P. Mestre, trads.). Revista Enunciación 15, 103-122.

Grice, P. (1975). Logic and Conversation En: Syntax and Semantics 3; Speech Acts. Cole and J.L Morgan, Academic Press. Traducido por Julio César Mejía en Lenguaje y sociedad (1973) Universidad del Valle, Cali, p. 101-121.

Gutiérrez, Y. (2009). La enseñanza y el aprendizaje de la oralidad en la educación media. En M. E. Rodríguez (comp.), Reflexiones y experiencias de investigación sobre la oralidad y la escritura (pp. 35-52). Bogotá: Universidad Distrital Francisco José de Caldas.

Gutiérrez, Y. (2011). Las concepciones disciplinares y didácticas de los profesores de lengua castellana sobre la lengua oral. En R. Pinilla y Y. Gutiérrez (comps.), La oralidad en contextos diversos: aportes investigativos para su discusión y comprensión (pp. 559-575). Bogotá: Universidad Distrital Francisco José de Caldas.

Jaimes, G. (1994). El desarrollo de la conciencia discursiva y su incidencia sobre los procesos de la lectura y la escritura. Bogotá: Universidad Externado de Colombia. 
74 Raquel Pinilla Vásquez, María Elvira Rodríguez Luna, Yolima Gutiérrez Ríos,

María Fernanda Torres, Martha Judith Camelo, Julia Esther Casjulia

Ministerio de Educación Nacional (MEN). (1998). Lineamientos curriculares. Bogotá: MEN.

Ministerio de Educación Nacional (MEN). (2003). Estándares curriculares. Bogotá: MEN.

Núñez, P. (2003). Didáctica de la comunicación oral: bases teóricas y orientaciones metodológicas para el desarrollo de la competencia discursiva oral en la educación obligatoria. Granada: Grupo Editorial Universitario.

Palou, J. y Bosch, C. (coords.).. (2005). La lengua oral en la escuela. 10 experiencias didácticas. Barcelona: Graó.
Pinilla, R. y Rodríguez, M. E. (2012). La evaluación de la oralidad. Un reto y un compromiso. Ponencia presentada en el XIII Congreso de la Sociedad Española de la Lengua y de la Literatura (SEDLL), Cádiz, España.

Porlán, R. y Rivero, A. (1998). El conocimiento de los profesores. Sevilla: Diada.

Riestra, D. (2008). Las consignas de trabajo en la escuela, un instrumento didáctico. Revista Novedades Educativas (Centro Regional Bariloche de la Universidad Nacional del Comahue), 211, 175-183.

Rodríguez Luna, M. E. (2006). Consideraciones sobre el discurso oral en el aula. Revista Enunciación 11, 59-72. 